\title{
Do clinical balance measures have the ability to predict falls among ambulatory individuals with spinal cord injury? A systematic review and meta-analysis
}

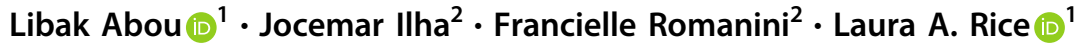 \\ Received: 25 March 2019 / Revised: 9 July 2019 / Accepted: 29 July 2019 / Published online: 2 September 2019 \\ (c) The Author(s), under exclusive licence to International Spinal Cord Society 2019
}

\begin{abstract}
Study design Systematic review and meta-analysis.

Background and purpose Fall prevalence is high among individuals with spinal cord injury (SCI) and falls may lead to serious consequences. The objective of this study was to investigate the ability of clinical balance measures to predict falls among ambulatory individuals with SCI.

Methods We searched the online databases MEDLINE, Web of Science, Scopus, SportDiscuss, and CINAHL. Two reviewers independently selected prospective and cross-sectional studies of ambulatory adults with SCI, with a method of falls tracking and a clinical balance evaluation. Risk of bias was assessed using the Newcastle-Ottawa Quality Assessment Scale. A meta-analysis of prospective and cross-sectional studies was performed using Review Manager 5.3.

Results Ten studies from the 2672 studies identified were included in the qualitative synthesis. Nine studies were included in the meta-analyses. The Berg Balance Scale (BBS) and the Timed Up and Go Test demonstrate ability to differentiate between fallers and non-fallers, mean difference 5.25 (95\% CI, 0.29 to 10.20$)$ and 6.65 (95\% CI, 0.17 to 13.12) respectively. The BBS presents moderate predictive ability (area under the receiver operating characteristic curve of 0.61 and sensitivity of $65 \%$ ). Individuals with a BBS score $\geq 40$ are likely at risk of falls.

Conclusion The BBS is the most appropriate and specific clinical balance measure with the ability to discriminate between ambulatory fallers and non-fallers. However, the fall predictive abilities of the measure need to be further explored and improved for this population.
\end{abstract}

\section{Introduction}

Falls are a common concern for individuals with spinal cord injury (SCI). The prevalence of falls is high among individuals who ambulate and among those who are nonambulatory. A previous study reported that within a

Supplementary information The online version of this article (https:// doi.org/10.1038/s41393-019-0346-5) contains supplementary material, which is available to authorized users.

Laura A. Rice

ricela@illinois.edu

1 Department of Kinesiology and Community Health, College of Applied Health Sciences, University of Illinois at UrbanaChampaign, Urbana, IL, USA

2 Programa de Pós-graduação em Fisioterapia, Universidade do Estado de Santa Catarina, Florianópolis, SC, Brazil reporting period of 12 months, $75 \%$ of ambulatory individuals with incomplete SCI experience one fall [1], most commonly while walking. A significant portion of falls result in physical injuries, including bruising [2], fractures, and concussion. These injuries often are sufficiently serious to require medical attention, resulting in increased assistance with activities of daily living and health care cost [3]. In addition, falls may lead to fear of falling, causing loss of independence, reduced mobility, depression, limitation in daily life activities, and restriction in social participation [4]. Therefore, the identification of individuals at risk of falls is crucial to develop effective fall prevention tools and reduce the subsequent consequences.

While there are many factors that influence or constitute fall risk among individuals with SCI, including muscle strength impairments [1, 5], performance of transfers, reaching activities, and environmental hazards [6,7], balance is commonly reported as one of the most important [6, 8-10]. In a clinical setting, a clinically feasible 
assessment measure of functional balance is required for monitoring changes in balance control [11] and for predicting falls. Several balance measures have been used as screening tests for fall risks among neurologically intact older adults [12, 13] and several neurological populations such as people with SCI, stroke, and multiple sclerosis [14]. A combination of the Berg Balance Scale (BBS) and a history of falls questionnaire showed good predictive abilities (91\% sensitivity and $82 \%$ specificity) and has been recommended as a predictive tool among older adults [13]. Similarly, the Timed Up and Go (TUG) Test has been recommended as a screening tool for fall risk and a measure of functional mobility by the American Society and British Society of Gerontology Guidelines [12]. Despite increased research on falls prediction in the SCI population in the past 5 years, little is known regarding the optimal balance performance cutoff between fallers and non-fallers, the clinical utility and discriminant ability of balance measures to predict falls in this population.

Thus, the purpose of this systematic review and metaanalysis was to investigate the ability of clinical balance measures to differentiate between ambulatory fallers and non-fallers with SCI. The sensitivity and specificity of the various measures were explored. We hypothesized there would be a significant difference between clinical balance test performance of fallers and non-fallers.

\section{Methods}

\section{Protocol and registration}

The review methodology protocol is registered in PROSPERO (CRD42018092026), Center for Reviews and Dissemination, University of York. The recommendations of the Preferred Reporting Items for Systematic Reviews and Meta-analyses (PRISMA) statement were followed throughout this study to strengthen and guide the conduct and reporting [15]. This study follows the strategy used in a previous systematic review and meta-analysis, which investigated the ability of clinical balance measures to predict fall risk in multiple sclerosis [16].

\section{Search strategy}

A systematic literature search was performed by the primary author (LA) in February 2018 and 15th May, 2019 and included the following databases: CINAHL, PubMed, SportDiscus (through EBSCO search platform), Web of Science and Scopus. These databases were chosen for providing literature in medicine, nursing and the health care system relevant to the topic of our study. Suitable keywords were identified by examining the search strategy used in other systematic reviews in this field $[11,16]$ and through discussions among the study authors and a librarian specialist in biomedical search strategy. The keywords and MeSH headings utilized as search terms were (spinal cord injuries OR SCI) AND (postural balance OR balance OR dynamic balance) AND (outcome assessment OR clinical measure) AND (fall OR fall risk). The literature search was supplemented by examining reference lists of identified articles. The full and detailed search strategy is available in Supplementary Appendix 1.

\section{Eligibility criteria}

Inclusion criteria were as follows: peer-reviewed published studies of ambulatory adults with SCI ( $>18$ years), use of a clinical balance measure, and a method of identifying and reporting falls. A clinical balance measure is defined as a standardized test or measure that is commonly used in clinical settings to monitor changes in a characteristic of interest during an episode of care [14]. Prospective cohort studies were included where balance measures were administered before a subsequent falls event, and falls were tracked prospectively using a fall calendar with regular phone calls or text messages to remind participants. Cross-sectional studies were included where participants were interviewed about their previous falls during a specific period of time before participating in the balance assessment. Studies with fall definitions such as "an unexpected event in which an individual comes to rest on the ground, floor, or lower level" [17] were included. The definition of a fall varied across the included studies. A faller was defined as an individual with a record of at least one fall during a period of 6 months or a year. Studies classifying an individual with $<2$ falls as an infrequent faller and an individual with two or more falls as recurrent fallers were also included. An ambulatory individual was defined as an individual with the ability to walk independently with or without walking aids, for $>75 \%$ of the time for mobility needs [18]. No language restriction was applied. Case reports were excluded as well as studies where laboratory-based balance measures only were used (e.g., posturography using force platforms, limits of stability using the Smart Balance Master). Studies including a mixed neurological population, redundant data, combined use of retrospective and prospective fall tracking or performance of dual-task assessments were excluded. We considered a dual-task assessment a concurrent assessment of balance and cognitive capacity (e.g., TUG + Stroop color and Word Test). Studies including dual-task were excluded because it is difficult to isolate the effect of the cognitive test and the balance performance. 


\section{Study selection}

Two independent reviewers (LA and FR) read the titles and/or abstracts of the identified studies and discarded irrelevant studies based on the inclusion and exclusion criteria. Potential studies for inclusion were read in full, and their suitability for inclusion was determined independently by two reviewers (LA and FR). Where disagreement on eligibility for inclusion occurred, discussion took place until consensus was reached. A list of Full-text excluded studies with detailed reasons can be found in Supplementary Appendix 2.

\section{Data extraction}

Study details including author name, year of publication, design, eligibility criteria, participant characteristics, balance measures, study specific fall definition, and how participants were classified related to fall frequency (e.g., faller, non-faller, recurrent faller, infrequent faller) were extracted to provide summary tables. A separate data extraction sheet was developed for prospective and cross-sectional studies. One researcher (LA) extracted the data, and a second (JI) checked the data against the full-text papers to enhance the rigor of data extraction. Study authors were contacted to provide supplemental information on study details when insufficient data were provided in the full-text.

\section{Quality assessment}

Two of the authors (LA and FR) independently evaluated the risk of bias of the included studies. Discordance was resolved through discussion with a third reviewer (JI). This assessment was performed using the Newcastle-Ottawa Quality Assessment Scale (NOQAS) [19] for prospective cohort studies; NOQAS for case control studies was adapted to evaluate the cross-sectional studies. According to NOQAS, each study is assessed based on three broad perspectives: the selection of the study groups, the comparability of the groups, and the exposure or outcome of interest. A star system was used to allow a semi-quantitative assessment of study quality. A maximum score of ten stars may be given [19]. The quality of the studies is rated according to the following criteria:[19]

Good quality: three or four stars in the selection domain AND one or two stars in the comparability domain AND two or three stars in the outcome/exposure domain.

Fair quality: two stars in the selection domain AND one or two stars in the comparability domain AND two or three stars in the outcome/exposure domain.

Poor quality: zero or one star in the selection domain OR zero stars in the comparability domain OR zero or one stars in the outcome/exposure domain.

\section{Statistical analysis}

Meta-analyses were performed to compute the pooled mean difference in balance test outcomes between fallers and nonfallers using a $95 \%$ confidence interval (CI). Heterogeneity was quantified using $I^{2}$ statistics. $I^{2}$ values between 50 and $75 \%$ were considered to indicate moderate heterogeneity, $I^{2}$ values $>75 \%$ were considered to indicate high heterogeneity [20]. A random-effects modeling (REM) approach was used instead of the fixed-effects modeling (FEM) when the pooled data had moderate or high heterogeneity. A FEM was used when $I^{2}<50 \%$. To reduce the heterogeneity of the studies, reports comparing fallers with non-fallers and infrequent fallers with recurrent fallers were separately pooled. The pooled odds ratios are represented by a solid diamond at the bottom of the forest plot. The meta-analysis was conducted using the software Review Manager 5 (version 5.3, The Cochrane Collaboration) [21]. Mean scores of the groups, standard deviation, and sample size were extracted from the original articles. When median (Q2) and first (Q1) and third (Q3) quartiles were reported, the formula developed by Wan et al. [22]. was used to estimate the mean and the standard deviation:

$x \approx \frac{\mathrm{Q} 1+\mathrm{Q} 2+\mathrm{Q} 3}{3}$

$x$ : mean, Q1 : first quartile, Q3 : third quartile

$\mathrm{SD} \approx \frac{\mathrm{Q} 3-\mathrm{Q} 1}{\eta(\mathrm{n})}$

SD: standard deviation, Q1: first quartile, Q3: third quartile, $\eta$ (n): a function that depends on the sample size. Wan et al. [22] provided estimated constant values of the function $\eta(n)$ depending on the sample size, which were used to determine the SD.

The cutoff scores, discriminant ability, and clinical utility of the balance measures were also reported when available. The clinical utility of the balance measures was estimated using sensitivity and specificity. Sensitivity informs the clinician how good a balance measure is at correctly identifying a faller [23]. Specificity informs the clinician how good the balance measure is at correctly identifying a non-faller [23]. The closer the sensitivity or specificity is to $100 \%$, the more sensitive or specific the balance measure [24]. The discriminant ability of a measure to differentiate between faller and non-faller was reported when an area under the receiver operating characteristic curve (AUC) was determined in the studies. The discriminant ability was judged according to the following criteria: values between 0.5 and 0.6 indicate chance discrimination, values between 0.6 and 0.7 indicate moderate discrimination, values between 0.7 and 0.9 indicate good discrimination and a value of 1 indicates perfect discrimination [25]. 
Fig. 1 PRISMA study selection flow

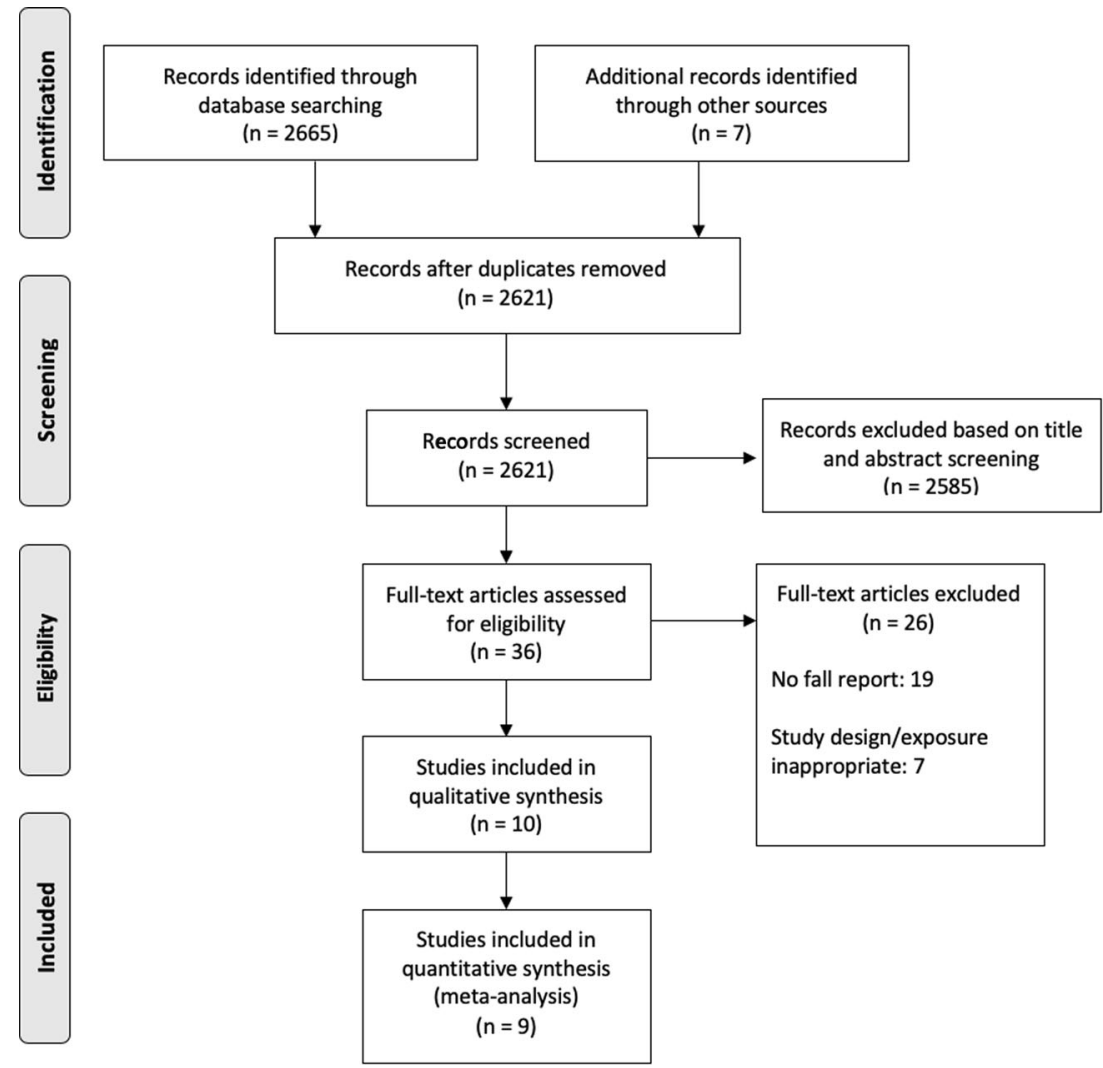

Quality assessment

Table 1a, b provide a summary of the quality assessment of the prospective cohort studies and cross-sectional studies, respectively. The NOQAS for prospective cohort studies showed that all the prospective studies had good methodological quality. Therefore, the prospective cohort studies presented a medium to low risk of bias (six to nine stars) $[2,9,10,18,27,28]$. The NOQAS for cross-sectional studies showed that all the cross-sectional studies had a poor methodological quality (five or six stars) indicating a high risk of bias.

\section{Meta-analysis of prospective studies}

Five studies used the TUG Test to compare fallers and nonfallers [9, 10, 27-29] (Fig. 2). Fallers required more time to complete this Test than non-fallers, showing significantly lower balance performance, REM, MD $=6.65 \mathrm{~s}, 95 \% \mathrm{CI}$ ( 0.17 to 13.12 ), $p<0.05, I^{2}=57 \%$, see Fig. $2 \mathrm{a}$. A randomeffects model was used for this meta-analysis due to the moderate heterogeneity observed.

The BBS was used twice in the prospective studies $[2,9]$. Surprisingly, the meta-analysis showed that fallers had significantly higher balance scores, FEM, MD $=5.25,95 \%$ CI $(0.29$ to 10.20$), p<0.05, I^{2}=0 \%$, see Fig. $2 b$. The BBS was also used to compare infrequent fallers and recurrent 


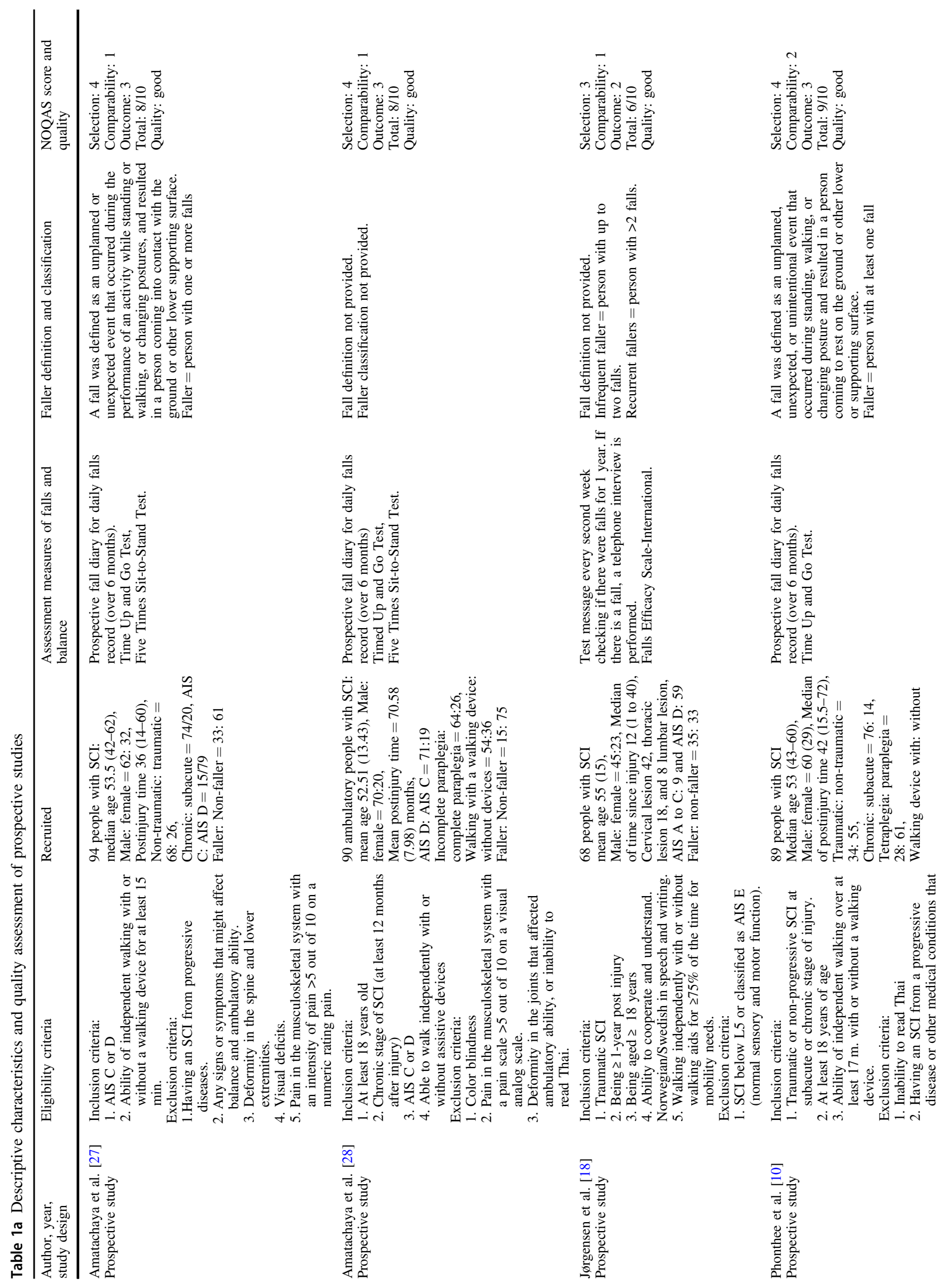




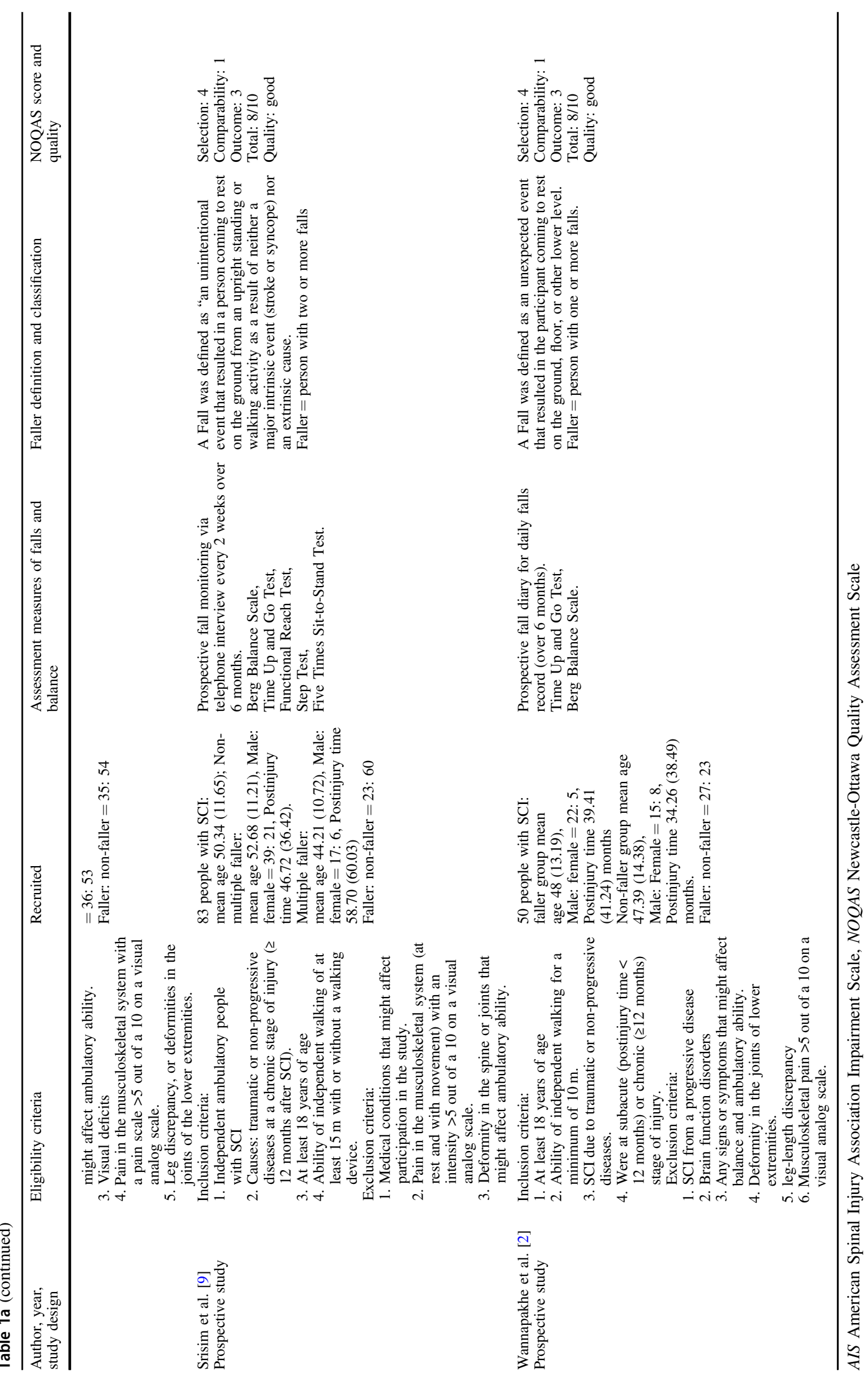




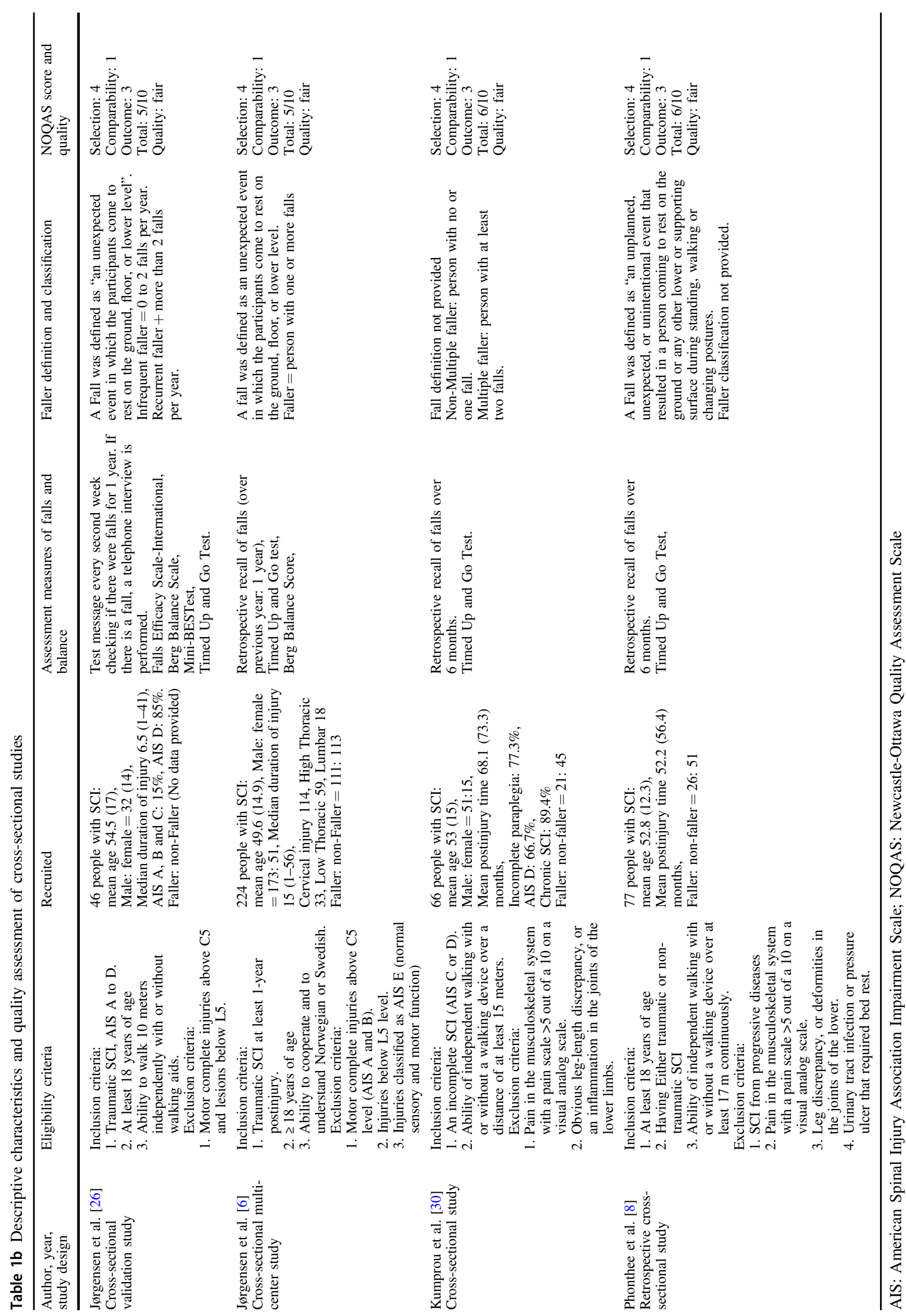


Table 2 Summary of the participant characteristics, falls duration, and balance measures included in the prospective and cross-sectional studies

\begin{tabular}{|c|c|c|c|}
\hline Study design & Participant characteristics & Falls duration & Balance measures \\
\hline \multicolumn{4}{|c|}{ Prospective studies (6) } \\
\hline & $\begin{array}{l}\text { Age range: } 42-62 \text { years } \\
\text { Range of participants: } 50-90 \\
\text { Total number of participants: } 474\end{array}$ & 6 months-1 year & $\begin{array}{l}\text { Timed Up and Go Test } \\
\text { Berg Balance Scale } \\
\text { Five Times Sit-to-Stand Test } \\
\text { Functional Reach Test } \\
\text { Step Test } \\
\text { Falls Efficacy Scale-International }\end{array}$ \\
\hline \multicolumn{4}{|c|}{ Cross-sectional studies (4) } \\
\hline & $\begin{array}{l}\text { Age range: } 49.6 \text { to } 54.5 \text { years } \\
\text { Range of participants: } 46 \text { to } 226 \\
\text { Total number of participants: } 413\end{array}$ & 6 months-1 year & $\begin{array}{l}\text { Timed Up and Go Test } \\
\text { Berg Balance Scale } \\
\text { Falls Efficacy Scale-International } \\
\text { Mini-BESTest }\end{array}$ \\
\hline
\end{tabular}

\section{Prospective}

\section{(a) Timed Up and Go test}

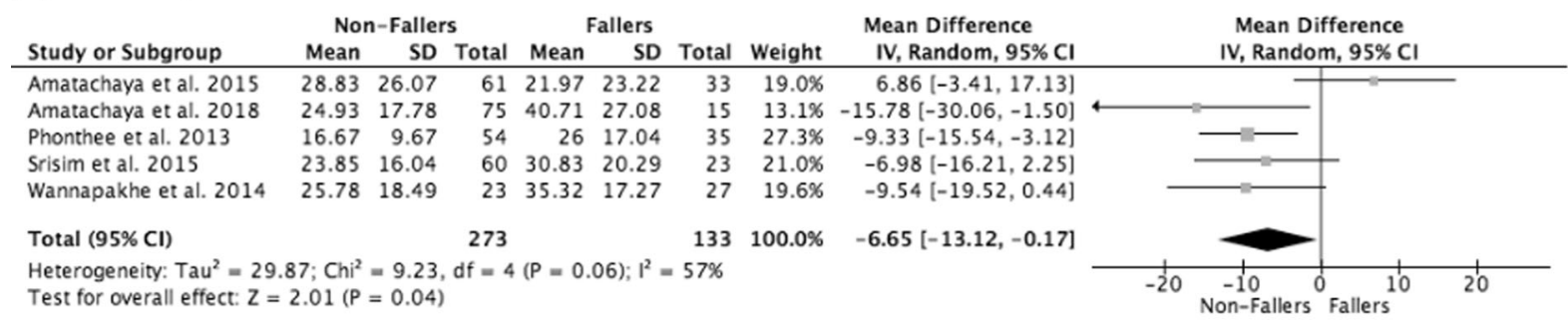

\section{(b) Berg Balance Scale}

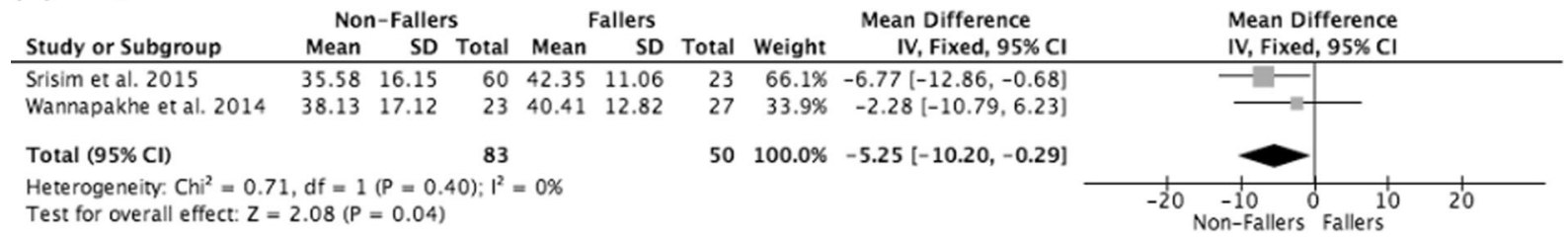

\section{(c) Five Times Sit-to-Stand}

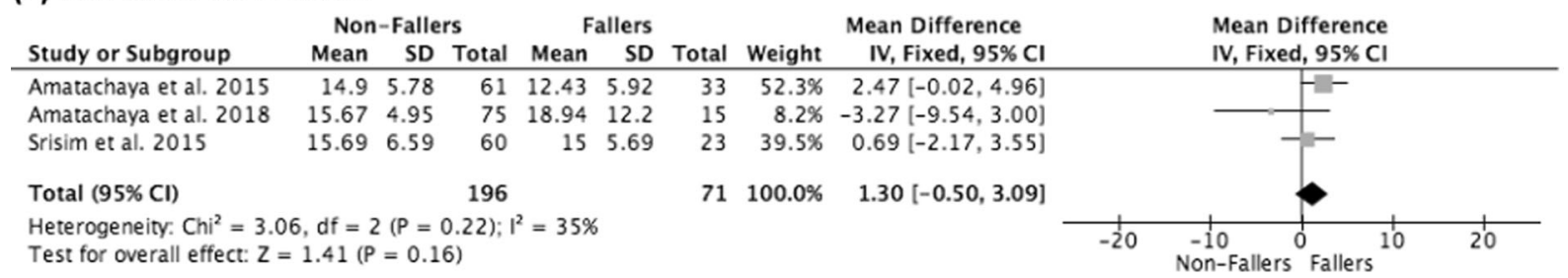

Fig. 2 Prospective studies: a Mean difference in Timed Up and Go test between non-fallers and fallers; $\mathbf{b}$ Mean difference in Berg Balance Scale between non-fallers and fallers; $\mathbf{c}$ Mean difference in Five Times sit-to-stand Test between non-fallers and fallers

fallers in another study [18]. There was no difference between the two groups in balance scores, $95 \% \mathrm{CI}(-0.73$ to 6.07$), p=0.12$.

Three prospective studies included in this review used the Five Times Sit-to-Stand (5TSTS) Test to compare fallers and non-fallers $[9,27,28]$. The result of the meta-analysis showed that there was no significant difference between fallers and non-fallers, $95 \% \mathrm{CI}(-0.5$ to 3.09$), p>0.05$, see Fig. 2c.
The Functional Reach (FR) Test [9] and the Step Test [9] were only used in one prospective study. Surprisingly, Srisim et al. [9] reported that non-fallers performed significantly lower on the FR Test than fallers (Mean difference $=6.4 \mathrm{~cm}, p<0.05$ ). There was no difference between fallers and non-fallers for the Step Test [9].

The FES-I [18] was also used in only one prospective study. Recurrent fallers showed a statistically significant 


\section{Cross-sectional}

(a) Timed Up and Go test

\begin{tabular}{|c|c|c|c|c|c|c|c|c|c|}
\hline \multirow[b]{2}{*}{ Study or Subgroup } & \multicolumn{3}{|c|}{ Non-Fallers } & \multicolumn{3}{|c|}{ Fallers } & \multicolumn{2}{|r|}{ Mean Difference } & \multirow{2}{*}{$\begin{array}{l}\text { Mean Difference } \\
\text { IV, Fixed, } 95 \% \mathrm{Cl}\end{array}$} \\
\hline & Mean & SD & Total & Mean & SD & Total & Weight & IV, Fixed, 95\% CI & \\
\hline Kumprou et al. 2017 & 30.61 & 22.08 & 45 & 28.12 & 19.9 & 21 & $16.1 \%$ & $2.49[-8.19,13.17]$ & \\
\hline Phonthee et al. 2013 & 23.9 & 13 & 51 & 16.3 & 7.9 & 26 & $83.9 \%$ & $7.60[2.91,12.29]$ & \\
\hline Total $(95 \% \mathrm{CI})$ & & & 96 & & & 47 & $100.0 \%$ & $6.78[2.48,11.07]$ & \\
\hline \multicolumn{9}{|c|}{$\begin{array}{l}\text { Heterogeneity: } C h i^{2}=0.74, d f=1(P=0.39) ; 1^{2}=0 \% \\
\text { Test for overall effect: } Z=3.10(P=0.002)\end{array}$} & 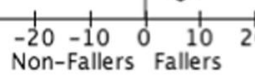 \\
\hline
\end{tabular}

(b) Timed up and Go test

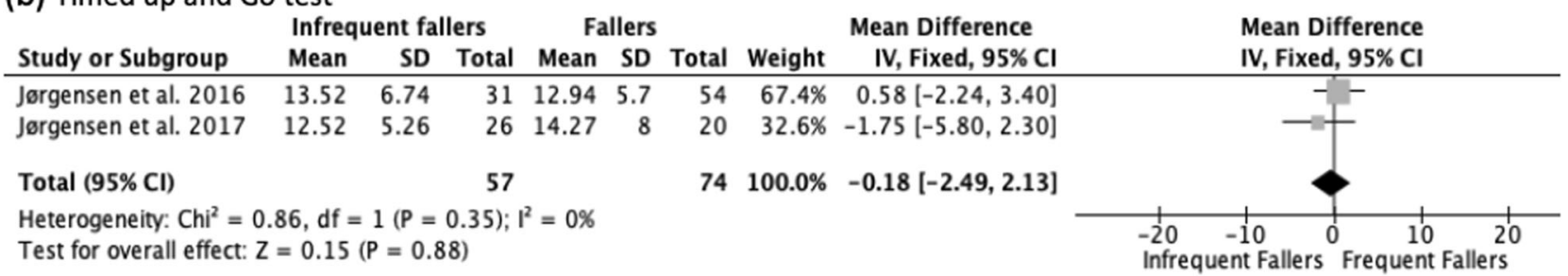

(c) Berg Balance Scale

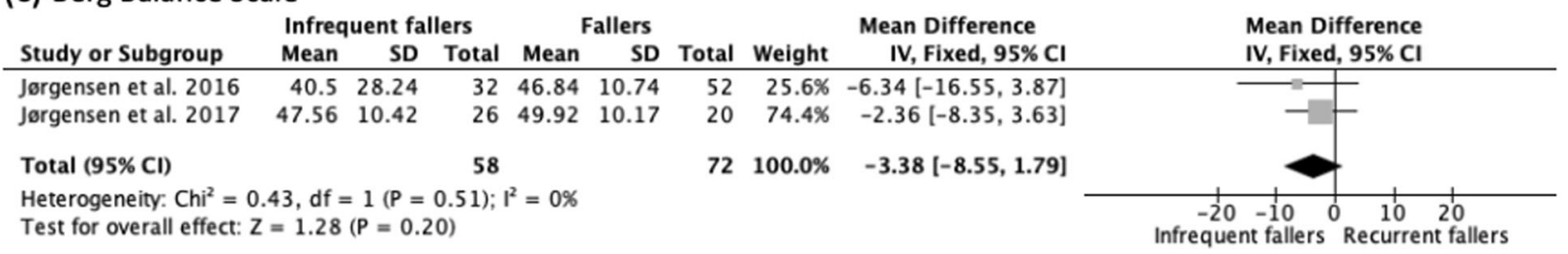

Fig. 3 Cross-sectional studies: a Mean difference in Timed Up and Go test between non-fallers and fallers; $\mathbf{b}$ Mean difference in Timed Up and Go test between infrequent fallers and recurrent fallers; $\mathbf{c}$ Mean difference in Berg Balance Scale between infrequent fallers and recurrent fallers

higher concern about falling than infrequent fallers (Mean difference $=16$ points, $p<0.01)[18]$.

\section{Meta-analysis of cross-sectional studies}

The results of the meta-analysis of two studies [8, 30] showed that the TUG Test was able to differentiate between fallers and non-fallers (Fig. 3). Fallers required less time to complete the Test. Surprisingly, non-Fallers demonstrated significantly lower balance performance, $\mathrm{FEM}, \mathrm{MD}=6.78$ s, $95 \%$ CI (2.48 to 11.07$), p<0.01, I^{2}=0 \%$, see Fig. 3 a. These results should be interpreted with caution as only two studies were included, and both were rated as having high risk of bias.

Two cross-sectional studies compared infrequent fallers and recurrent fallers using the TUG Test and the BBS $[6,26]$. The meta-analyses showed that these scales were not able to discriminate between the groups, 95\% CI (2.49 to 2.13$), p=0.88$ and $95 \%$ CI $(-8.55$ to 1.79$)$, $p=0.20$ respectively, see Fig. $3 \mathrm{~b}$, c.

The Mini-BESTest was only used in one cross-sectional study. There was no difference between infrequent fallers and recurrent fallers, 95\% CI $(-4.51$ to 5.33$), p=0.87$. The FES-I was the only self-report measure used to compare infrequent and recurrent fallers [26]. The comparison showed there was no difference between recurrent fallers and infrequent fallers in concerns about falling $(p=0.36)$.

\section{Discriminant ability and clinical utility of the measures}

Two studies $[9,26]$ included in this review investigated the clinical utility of six balance measures to predict falls. The results are summarized in Table 3. Overall, all the measures showed only a moderate discrimination ability. In addition, none of the measures presented a sensitivity higher than $80 \%$. A cutoff score of at least $20 \mathrm{~cm}$ for the FR Test was reported by Srisim et al. [9] as the best indicator of risk of falls, sensitivity $=73 \%$, specificity $=55 \%$, and AUC $=0.64$.

\section{Discussion}

The purpose of this systematic review and meta-analysis was to evaluate the association between clinical balance measures and falls among ambulatory individuals with SCI. Overall, few studies analyzed this association using clinical balance measures. The BBS, the TUG Test and the 5TSTS Test were the most commonly administered. Based on the pooled results from the meta-analysis, the BBS and the 
Table 3 Clinical utility and discriminative ability of clinical balance measures

\begin{tabular}{|c|c|c|c|c|c|}
\hline Study ID & Clinical measure & AUC value & Sensitivity (\%) & Specificity (\%) & Cutoff value \\
\hline \multicolumn{6}{|c|}{ Prospective study } \\
\hline \multirow{5}{*}{$\begin{array}{l}\text { Srisim } \\
\text { et al. [9] }\end{array}$} & Berg Balance Scale & 0.61 & 65 & 53 & $\geq 40$ \\
\hline & $\begin{array}{l}\text { Timed Up and } \\
\text { Go test }\end{array}$ & 0.57 & 56 & 68 & $\geq 26 \mathrm{~s}$ \\
\hline & $\begin{array}{l}\text { Functional } \\
\text { Reach test }\end{array}$ & 0.64 & 73 & 55 & $\geq 20 \mathrm{~cm}$ \\
\hline & Step Test & 0.36 & 44 & 48 & $\geq 13 \mathrm{~s}$ \\
\hline & $\begin{array}{l}\text { Fives Times Sit-to- } \\
\text { Stand Test }\end{array}$ & 0.48 & 50 & 50 & $\geq 15 \mathrm{~s}$ \\
\hline \multicolumn{6}{|c|}{ Cross-sectional study } \\
\hline \multirow[t]{2}{*}{$\begin{array}{l}\text { Jørgensen } \\
\text { et al. [26] }\end{array}$} & Berg Balance Scale & $\begin{array}{l}0.52 \\
(0.36-0.69)\end{array}$ & $0.55(0.32-0.77)$ & $0.50(0.30-0.70)$ & $>50$ \\
\hline & Mini-BESTest & $\begin{array}{l}0.54 \\
(0.36-0.69)\end{array}$ & $0.55(0.31-0.77)$ & $0.54(0.33-0.73)$ & $>19$ \\
\hline
\end{tabular}

The $95 \%$ confidence interval is reported in brackets after the value

$A U C$ area under the receiver operating characteristic curve statistic, $\mathrm{cm}$ centimeter, $s$ second
TUG Test demonstrate ability to discriminate between fallers and non-fallers. The sensitivity and specificity reported for these measures were fair. In addition, a fair discriminative ability was reported for the TUG Test and a moderate discriminative ability was found for the BBS, AUC of 0.57 and 0.61 respectively. Therefore, the BBS presents greater ability to discriminate between fallers and non-fallers. The BBS evaluates walking balance exclusively while the TUG Test assesses walking balance, strength, coordination, and ambulation ability. Thus, the TUG Test is a functional mobility measure and not a pure balance measure [14]. Recently, Moore et al. [14] suggested the BBS as the only static and dynamic sitting and standing balance measure with appropriate measurement properties to be used for adults with acute, subacute and chronic neurological conditions. Also, the FR Test is a single-item test included as an item in the BBS and presents good discriminative ability and good sensitivity (see Table 3). The analysis of the studies included in this investigation showed that the BBS presents the appropriate characteristics to be used as a fall prediction balance measure for ambulatory individuals with SCI. However, further prospective studies are needed to improve the falls prediction properties of the measure.

The meta-analysis of the prospective studies showed a significant difference between fallers and non-fallers in the TUG Test and BBS scores. The mean difference of $6.65 \mathrm{~s}$ found between fallers and non-fallers for the TUG Test in our study is smaller than the Smallest Real Difference (SRD) of $10.8 \mathrm{~s}$ established for this measure [31]. However, the mean difference of 5.25 found between fallers and non-fallers for the BBS is similar to the SRD reported for other populations since the SRD of the BBS is not yet established for individuals with SCI. Previous studies reported a SRD of 5 points for individuals with Parkinson [32] and 4.66 for individuals with chronic stroke [33]. In addition, for the TUG Test, fallers demonstrated worse balance, while on the BBS, fallers had better balance scores. The conflicting result is also reported in a recent systematic review [34]. Some authors argue that among ambulatory individuals with SCI, a good functional ability is related to an exposure to a risk of falls [8, 28, 30], while other authors report that falls are related to lower functional ability [2, 9, 10]. A possible explanation for the findings may be because falls are multifactorial. Falls depend on several factors and circumstances [6, 7, 35], including: the type of activities the individual performs, environmental hazards the person encounters, the quantity of aids (e.g., canes, walkers) the individual uses, her or his confidence level, safety awareness, the individual's energy level (e.g., higher level of energy may increase the attempts of performing more activities), and the attention to movements. The biological or physical factors contributing to falls such as impaired balance, muscle weakness, and spasticity are normally targeted in clinical settings to improve functionality and reduce falls frequency. In addition, other modifiable risk factors should be considered in attempt to reduce falls frequency. Behavioral factors (e.g., inattention or distraction during movement, fear of falling, not using protective straps or safety belt, taking risks) and environmental factors (e.g., uneven and slippery surfaces, cluttered environment) should therefore be considered in developing SCI-specific fall prevention programs [34]. Also, a study reported falls in the previous year was a strong predictor of future falls among individuals with SCI [36]. The single-item question of falls in 
the previous year can be easily obtained in clinical settings via a semi-structured interview. The single-item question can be used together with the BBS to predict future falls among this population.

The clinical utility reported in the studies demonstrated that all the clinical balance measures have a sensitivity lower than $80 \%$. Even though the BBS and the FR Test showed higher and promising clinical utilities to predict falls (see Table 3), all the clinical balance measures have an AUC lower than 0.7 indicating these measures have a moderate ability to discriminate between fallers and nonfallers. The BBS includes the FR Test as an item. The clinical utility to predict falls reported for the BBS may be moderate because balance assessment by itself is not enough to fully predict falls among ambulatory individuals with SCI. Future prospective studies are warranted to explore the clinical utility of a combination of the BBS and other functional measures to predict falls. The results of this systematic review are similar to the findings reported for individuals with multiple sclerosis, Parkinson disease and older adults, which suggest further prospective studies are required to improve the clinical utility of balance measures to predict falls [16, 37, 38].

Since falls are multifactorial, it is not surprising that many of the balance measures were not diagnostic on their own. The complexity of falls may also justify the contradictory findings on balance performance among the measures analyzed. In addition, falls reporting in cross-sectional studies is based on participants' memory and may not be reliable. However, prospective studies use fall calendar tracking accompanied by regular phone calls or text messages to remind participants. Therefore, prospective cohort studies should be prioritized over cross-sectional studies. We recommend that the results of this systematic review and meta-analysis be used to inform further studies to improve the abilities of clinical balance measures to predict falls. For example, among all the identified measures, the FR Test presents the best discriminant ability and sensitivity with a cutoff value greater or equal to $20 \mathrm{~cm}$ [9] and the BBS moderately well differentiates fallers and non-fallers (cutoff score greater or equal to 40). The BBS presents the appropriate characteristics to predict falls since it integrates the several components assessment of balance including the FR Test.

We were also expecting to identify clinical balance measures able to predict falls among non-ambulatory individuals with SCI. Insufficient data were available to analyze the topic. Therefore, we limited our review to the ambulatory population. Since the prevalence of falls is also high among non-ambulatory individuals [39], we suggest that future studies explore the ability of clinical balance measures to predict falls among non-ambulatory individuals with SCI.

\section{Limitations}

There are some limitations to this study. First of all, the meta-analysis was done with a small number of studies. This may weaken the interpretation of our results. In addition, the meta-analysis of the TUG Test for prospective studies presents a heterogeneity of $57 \%$.

Another limitation was the inclusion of measures that were not designed exclusively to assess balance, such as the TUG Test and the 5TSTS Test. Even though these measures evaluate balance, they are also considered measures of mobility and transfers, which may complicate the interpretation of the results. However, we decided to include the measures since they are widely used in clinical settings as balance measures, especially the TUG Test.

\section{Strength of the systematic review}

Faller classifications varied across the identified studies. Some studies classified fallers as individuals with one fall while others considered fallers as individuals with two or more falls. To minimize the effect of this heterogeneity, the comparisons of fallers VS non-fallers and infrequent fallers VS recurrent fallers were done separately.

Moreover, we used a standardized and well-organized methodological approach to perform the search, to select the included articles, to provide a list of excluded articles, to assess the risk of bias, to perform the meta-analyses, and to analyze the results. This strengthens our findings and reports on the current state of the ability of clinical balance measures to predict falls among individuals with SCI.

\section{Conclusion}

Based on the results of this systematic review and metaanalysis, it can be claimed that the BBS and the TUG Test differentiate between ambulatory fallers and non-fallers with SCI. The discriminative ability and the clinical utility to predict falls reported for the TUG and BBS were fair to moderate. However, the BBS appears to be the most appropriate clinical balance measure to be used for falls prediction ability among ambulatory individuals with SCI. A BBS score greater or equal to 40 identifies individuals who are at a greater risk of falls. The FR Test, which is an item included in the BBS, also presented good predictive ability. However, it is worthwhile to point out that the predictive ability of the FR Test has been investigated in only one study [9] and therefore, general conclusions cannot be drawn. Since falls are multifactorial, further prospective cohort studies are required to examine more in-depth the relationships between fall history, fear of falling, and the clinical balance or other mobility measures. This may 
improve the ability of the BBS to predict falls among ambulatory individuals with SCI.

Acknowledgements We would like to thank the librarian JJ Pionke for the assistance building the search strategy and the undergraduate students who helped in the preparation of this manuscript. We would also like to gratefully acknowledge the data contributed by the following researchers: Dr. Sugalya Amatachaya, Vivien Jørgensen, and Emelie Forslund.

Author contributions LA was responsible for designing the initial review protocol, registering the protocol, conducting the search, screening potentially eligible studies, extracting data, performing quality assessment of the included articles, interpreting results, and writing the initial manuscript. JI was responsible for assisting with eligible studies, reviewing data extraction, data analyses, interpreting results, and provided feedback on the manuscript. FR was responsible for screening potentially eligible studies, performing quality assessment of included articles. LR contributed to the study idea, provided feedback on the initial review protocol, on results interpretation, and on the manuscript.

\section{Compliance with ethical standards}

Conflict of interest The authors declare that they have no conflict of interest.

Publisher's note: Springer Nature remains neutral with regard to jurisdictional claims in published maps and institutional affiliations.

\section{References}

1. Brotherton SS, Krause JS, Nietert PJ. Falls in individuals with incomplete spinal cord injury. Spinal Cord. 2007;45:37-40.

2. Wannapakhe J, Arayawichanon P, Saengsuwan J, Amatachaya S. Changes of functional ability in patients with spinal cord injury with and without falls during 6 months after discharge. Phys Ther. 2014;94:675-81.

3. Krause JS. Factors associated with risk for subsequent injuries after traumatic spinal cord injury. Arch Phys Med Rehabil. 2004;85:1503-8.

4. WHO. World Health Organization Global Report on falls prevention in older age. 2017 [http://www.who.int/ageing/publica tions/Falls_prevention7March.pdf].

5. Amatachaya S, Wannapakhe J, Arrayawichanon P, Siritarathiwat W, Wattanapun P. Functional abilities, incidences of complications and falls of patients with spinal cord injury 6 months after discharge. Spinal Cord. 2011;49:520-4.

6. Jørgensen V, Butler Forslund E, Franzén E, Opheim A, Seiger Å, Ståhle A, et al. Factors associated with recurrent falls in individuals with traumatic spinal cord injury: a multicenter study. Arch Phys Med Rehabil. 2016;97:1908-16.

7. Musselman KE, Arnold C, Pujol C, Lynd K, Oosman S. Falls, mobility, and physical activity after spinal cord injury: an exploratory study using photo-elicitation interviewing. Spinal Cord Ser Cases. 2018;4:39.

8. Phonthee S, Saengsuwan J, Amatachaya S. Falls in independent ambulatory patients with spinal cord injury: incidence, associated factors and levels of ability. Spinal Cord. 2013;51:365-8.

9. Srisim K, Saengsuwan J, Amatachaya S. Functional assessments for predicting a risk of multiple falls in independent ambulatory patients with spinal cord injury. J Spinal Cord Med. 2015;38:439-45.
10. Phonthee S, Saengsuwan J, Siritaratiwat W, Amatachaya S. Incidence and factors associated with falls in independent ambulatory individuals with spinal cord injury: a 6-month prospective study. Phys Ther. 2013;93:1061-72.

11. Abou L, de Freitas GR, Palandi J, Ilha J. Clinical instruments for measuring unsupported sitting balance in subjects with spinal cord injury: a systematic review. Top Spinal Cord Inj Rehabil. 2018;24:177-93.

12. Panel on Prevention of Falls in Older Persons AeGSaBGS. Summary of the Updated American Geriatrics Society/British Geriatrics Society clinical practice guideline for prevention of falls in older persons. J Am Geriatr Soc. 2011;59:148-57.

13. Shumway-Cook A, Baldwin M, Polissar NL, Gruber W. Predicting the probability for falls in community-dwelling older adults. Phys Ther. 1997;77:812-9.

14. Moore JL, Potter K, Blankshain K, Kaplan SL, O'Dwyer LC, Sullivan JE. A core set of outcome measures for adults with neurologic conditions undergoing rehabilitation: a clinical practice guideline. J Neurol Phys Ther. 2018;42:174-220.

15. Moher D, Liberati A, Tetzlaff J, Altman DG, Group P. Preferred reporting items for systematic reviews and meta-analyses: the PRISMA statement. Brit Med J. 2009;339:b2535.

16. Quinn G, Comber L, Galvin R, Coote S. The ability of clinical balance measures to identify falls risk in multiple sclerosis: a systematic review and meta-analysis. Clin Rehabil. 2018:32:571-82.

17. Lamb SE, Jørstad-Stein EC, Hauer K, Becker C, Group PoFNEaOC. Development of a common outcome data set for fall injury prevention trials: the Prevention of Falls Network Europe consensus. J Am Geriatr Soc. 2005;53:1618-22.

18. Jørgensen V, Butler Forslund E, Opheim A, Franzén E, Wahman $\mathrm{K}$, Hultling $\mathrm{C}$, et al. Falls and fear of falling predict future falls and related injuries in ambulatory individuals with spinal cord injury: a longitudinal observational study. J Physiother. 2017;63:108-13.

19. Wells G, Shea B, O'Connell D, Peterson J, Welch V, Losos M, et al. The Newcastle-Ottawa Scale (NOS) for assessing the quality of non-randomized studies in meta-analyses [Available from: http://www.ohri.ca/programs/clinical_epidemiology/oxford.asp.

20. Higgins JP, Thompson SG. Quantifying heterogeneity in a metaanalysis. Stat Med. 2002;21:1539-58.

21. (RevMan) RM. [Computer Program]. Version 5.3 ed. Copenhagen: The Nordic Cochrane Centre, The Cochrane Collaboration; 2014.

22. Wan X, Wang W, Liu J, Tong T. Estimating the sample mean and standard deviation from the sample size, median, range and/or interquartile range. BMC Med Res Method. 2014;14:135.

23. Sackett DL. The rational clinical examination. A primer on the precision and accuracy of the clinical examination. J Am Med Assoc. 1992;267:2638-44.

24. Riddle DL, Stratford PW. Interpreting validity indexes for diagnostic tests: an illustration using the Berg balance test. Phys Ther. 1999;79:939-48.

25. Hajian-Tilaki K. Receiver operating characteristic (ROC) curve analysis for medical diagnostic test evaluation. Casp J Intern Med. 2013;4:627-35.

26. Jørgensen V, Opheim A, Halvarsson A, Franzén E, Roaldsen KS Comparison of the Berg Balance Scale and the Mini-BESTest for assessing balance in ambulatory people with spinal cord injury: validation study. Phys Ther. 2017;97:677-87.

27. Amatachaya S, Pramodhyakul W, Wattanapan P, Eungpinichpong W. Ability of obstacle crossing is not associated with falls in independent ambulatory patients with spinal cord injury. Spinal Cord. 2015;53:598-603.

28. Amatachaya S, Srisim K, Thaweewannakij T, Arrayawichanon P, Amatachaya P, Mato L. Failures in dual-task obstacle crossing 
could predict risk of future fall in independent ambulatory individuals with spinal cord injury. Clin Rehabil. 2018;269215518788913

29. Zarco-Periñan MJ, Barrera-Chacón MJ, García-Obrero I, MendezFerrer JB, Alarcon LE, Echevarria-Ruiz, et al. Development of the Spanish version of the Spinal Cord Independence Measure version III: cross-cultural adaptation and reliability and validity study. Disabil Rehabil. 2014;36:1644-51.

30. Kumprou M, Amatachaya P, Sooknuan T, Thaweewannakij T, Amatachaya S. Is walking symmetry important for ambulatory patients with spinal cord injury? Disabil Rehabil. 2018;40:836-41.

31. Lam T, Noonan VK, Eng JJ, Team SR. A systematic review of functional ambulation outcome measures in spinal cord injury. Spinal Cord. 2008;46:246-54.

32. Steffen T, Seney M. Test-retest reliability and minimal detectable change on balance and ambulation tests, the 36-item short-form health survey, and the unified Parkinson disease rating scale in people with parkinsonism. Phys Ther. 2008;88:733-46.

33. Hiengkaew V, Jitaree K, Chaiyawat P. Minimal detectable changes of the Berg Balance Scale, Fugl-Meyer Assessment Scale, Timed "Up \& Go" Test, gait speeds, and 2-minute walk test in individuals with chronic stroke with different degrees of ankle plantarflexor tone. Arch Phys Med Rehabil. 2012;93:1201-8.
34. Khan A, Pujol C, Laylor M, Unic N, Pakosh M, Dawe J, et al. Falls after spinal cord injury: a systematic review and metaanalysis of incidence proportion and contributing factors. Spinal Cord. 2019;57:526-39.

35. Sung J, Trace Y, Peterson EW, Sosnoff JJ, Rice LA. Falls among full-time wheelchair users with spinal cord injury and multiple sclerosis: a comparison of characteristics of fallers and circumstances of falls. Disabil Rehabil. 2019;41:389-95.

36. Butler Forslund E, Jørgensen V, Skavberg Roaldsen K, Hultling C, Wahman K, Franzén E. Predictors of falls in persons with spinal cord injury-a prospective study using the Downton fall risk index and a single question of previous falls. Spinal Cord. 2018;57:91-9.

37. Paul SS, Canning CG, Sherrington C, Lord SR, Close JC, Fung VS. Three simple clinical tests to accurately predict falls in people with Parkinson's disease. Mov Disord. 2013;28:655-62.

38. Barry E, Galvin R, Keogh C, Horgan F, Fahey T. Is the Timed Up and Go test a useful predictor of risk of falls in community dwelling older adults: a systematic review and meta-analysis. BMC Geriatr. 2014;14:14.

39. Forslund EB, Jørgensen V, Franzén E, Opheim A, Seiger A, Ståhle A, et al. High incidence of falls and fall-related injuries in wheelchair users with spinal cord injury: A prospective study of risk indicators. J Rehabil Med. 2017;49:144-51. 